\title{
Crenças e práticas educativas de mães de crianças com desenvolvimento atípico
}

\section{Beliefs and educational practices of mothers of children with atypical development}

\author{
Maria de Fátima Minetto ${ }^{1}$ \\ Suzane Schmidlin Löhr ${ }^{1}$
}

\begin{abstract}
RESUMO
O desenvolvimento humano atípico interfere no processo de aprendizado da criança que o apresenta, sendo que os pais têm importante papel no sentido de estimular o desenvolvimento dos filhos. Porém, o envolvimento paterno sofre influência das crenças que os pais têm sobre o desenvolvimento dos filhos. Para avaliá-las, Suizzo (2002) construiu a Escala de Crenças Parentais e Práticas de Cuidado (E-CPPC). Assim, na presente pesquisa foi realizada uma pesquisa descritiva e transversal, utilizando o E-CPPC com 75 mães de crianças com diagnóstico de deficiência intelectual, das quais 41 sem fenótipo (grupo que abrangeu, dentre outros, crianças com Transtorno do Espectro Autista), cuja deficiência intelectual foi percebida nos primeiros anos de vida, e 34 mães de crianças com Síndrome de Down, identificada quando do nascimento. Das crianças com Síndrome de Down, 94,11\% frequentavam escola especial e este percentual baixou para $80,48 \%$ nas crianças sem fenótipo. As mães dos dois agrupamentos apontaram em primeiro lugar a dimensão Apresentação Apropriada do bebê, envolvendo aqui a valorização de cuidados básicos, vindo a seguir: Estimulação; Responsividade/vínculo; Disciplina. Na análise de pares de dimensões do E-CPPC foi percebido que somente no par de dimensões Apresentação-Responsividade/vínculo houve diferença entre os grupos, sendo positiva e com significância estatística nas crianças com Síndrome de Down e não apresentando relação significativa no outro grupo. Poder contar com rede de apoio o quanto antes surge como sugestão importante aos dois grupos.
\end{abstract}

Palavras-chave: práticas de cuidado; práticas educativas; desenvolvimento atípico; crenças parentais.

DOI: $10.1590 / 0104-4060.44791$

1 Universidade Federal do Paraná, Setor de Educação. Curitiba, Paraná, Brasil. Rua General Carneiro, nº 460. CEP: 80060-150.E-mails: fa.minetto@gmail.com; lohr@superig.com.br 


\begin{abstract}
Atypical human development interferes in the learning process of children who have it and parents have an important role to play in stimulating the development of their children. However, parental involvement suffers the influence of the beliefs they have regarding the development of their children. In order to assess such beliefs Suizzo (2002) created the Scale of Parental Beliefs and Care Practices (S-PBCP). A descriptive cross-sectional study was conducted using S-PBCP with 75 mothers of children diagnosed as having intellectual disability, 41 of whom had no phenotype (among other disabilities, this group included children with Autistic Spectrum Disorders) and whose intellectual disability was perceived in the first years of life, as well as 34 mothers of children with Down Syndrome identified as such at birth. $94.11 \%$ of the children with Down Syndrome attended special schools, whilst this percentage fell to $80.48 \%$ in children with no phenotype. The first dimension indicated by the mothers in both groups was the Appropriate Presentation of the baby, involving the valuing of basic care, followed by: Stimulation; Responsiveness/bond; Discipline. When analysing pairs of $\mathrm{S}-\mathrm{PBCP}$ dimensions it was found that there was only a difference between the groups in the Presentation-Responsiveness/bond dimensions, it being positive and statistically significant for the children with Down Syndrome, but having no significant relationship for the other group. Being able to count on a support network as soon as possible appears as an important suggestion in both groups.
\end{abstract}

Keywords: care practices; educational practices; atypical development; parental beliefs.

\title{
Crianças com desenvolvimento atípico
}

O termo desenvolvimento atípico vem sendo utilizado para sinalizar pessoas com necessidades educativas especiais que apresentam deficiência física ou intelectual, ou prejuízo no acompanhamento do processo de ensino e aprendizagem por dificuldades e/ou deficiências que podem não estar vinculadas a uma causa orgânica, mas que requerem código de comunicação diferente do usado pela maioria dos estudantes. (CALLONERE; FERREIRA ROLIM; HÜBNER, 2011).

Em cada área do desenvolvimento humano existem padrões esperados que estão fundamentados na maturação biológica do indivíduo, mas sofrem influência da estimulação ambiental. O desenvolvimento não ocorrer segundo a norma 
caracteriza desenvolvimento atípico. Diversos fatores podem estar relacionados ao desenvolvimento atípico, sendo um deles os transtornos mentais na infância. Segundo Ebert, Lorenzini e Franco da Silva (2015), é estimado que, no Brasil, entre $10 \%$ e $20 \%$ de crianças e adolescentes apresentam transtornos mentais, dos quais, aproximadamente, de 3\% a 4\% necessitam de tratamentos intensivos.

Dentre os quadros de transtornos mentais na infância, o autismo é um dos mais frequentes. Crianças que apresentam Transtorno do Espectro Autista (TEA) têm desenvolvimento atípico na comunicação e interação social em múltiplos contextos, além de padrões restritos e repetitivos de comportamento, interesses ou atividades, aspectos considerados pelo Manual Diagnóstico e Estatístico de Transtornos Mentais (DSM-V) como condições para o estabelecimento do diagnóstico. (AMERICAN PSYCHIATRIC ASSOCIATION, 2014). Muitos aspectos que crianças em geral dominam de forma espontânea, crianças com TEA precisam que lhes seja ensinado de forma sistemática, estruturada. O papel da família, especialmente dos pais, é central nesse processo, mas as expectativas que eles têm sobre o desenvolvimento da criança irão interferir no seu modo agir.

Muitos estudos nos últimos 10 anos vêm investigando as expectativas do entorno das crianças com desenvolvimento atípico, tanto no convívio familiar quanto na inserção escolar: Matos et al. (2012) identificaram expectativas das mães sobre os filhos com Síndrome de Down; Leal (2006) teve como objetivo conhecer as expectativas das mães sobre a escolarização de seus filhos com Síndrome de Down; Gau et al. (2008) relacionaram características e estilos parentais a problemas de comportamento em crianças com Síndrome de Down e seus irmãos; Silva e Aiello (2009) descreveram características apenas dos pais de filhos com deficiência intelectual; Marques e Dixe (2011) analisaram o impacto na dinâmica familiar e pessoal de crianças e jovens com autismo; Minetto et al. (2012) relacionaram práticas educativas e estresse parental; Minetto, Crepaldi e Marins (2013) destacaram características de famílias com filhos com deficiência intelectual; Zanon, Backes e Bosa (2014) apontaram os pais como os primeiros a identificarem pautas de autismo nos filhos; Correa et al. (2015) descreveram fatores de risco ao desenvolvimento de crianças com Síndrome de Down. Também a busca de conhecimento sobre fatores de risco e de proteção ao desenvolvimento de crianças com deficiência ou transtornos globais vem aumentando o investimento em pesquisas nos últimos anos. (COUTO; TACHIBANA; AIELLO-VAISBERG, 2007; PEREIRA-SILVA; DESSEN, 2007; PRADO, 2005; SUNELAITIS; ARRUDA; MARCON, 2007).

A família, como o principal microssistema responsável pelo desenvolvimento da criança, na grande maioria das vezes, é o foco das investigações, uma vez que a chegada de um filho com alguma deficiência modifica planos, aumenta a responsabilidade e necessidades de investimentos, causa maior estresse e leva a 
questionamentos sobre as práticas educativas que os pais acreditavam ser ideais para sua prole. Os pais, além de prestadores de cuidados, modelos, disciplinadores e promotores da socialização dos filhos, assumem posição central no que se refere à estimulação inicial para o desenvolvimento dos filhos. (BRONFENBRENNER; MORRIS, 2006; PÉREZ-LÓPEZ et al., 2012; FRANCO, 2015).

Quando a criança apresenta alguma vulnerabilidade e encontra-se em risco de desenvolvimento atípico, modelos de intervenção centrados na família, focados no seu empoderamento, tornam-se alternativas produtivas e podem envolver preparo dos pais para apoio dos filhos por toda a vida. As famílias, além de informação, precisam aumentar sua competência para acessar os recursos da comunidade e também oportunizar atividades voltadas para a satisfação de necessidades físicas, habilidades sociais e culturais da criança. (COUTINHO, 2004; VIEIRA; PRADO, 2004).

Coutinho (2004) relata que em Portugal este tem sido um caminho lento e só recentemente os pais passaram a ser incluídos no atendimento profissional visando cuidado de seus filhos. Para avaliar os efeitos do empoderamento de mães de crianças com Síndrome de Down com idades entre seis meses e cinco anos, a autora desenvolveu estudo com 39 mães de crianças com Síndrome de Down, das quais 22 foram incluídas em um Programa de Formação de Pais de 12 semanas visando formar, informar e fornecer apoio social e as 17 mães restantes foram incluídas em grupo controle. Aconteceram importantes ganhos no que tange à percepção materna de competência - as mães descreveram ao final do programa que se sentirem mais aptas a estimular o desenvolvimento dos filhos desde que orientadas. Houve também ganho nos escores de autoeficácia materna.

Ebert, Lorenzini e Franco da Silva (2015), ao estudarem a percepção de mães de crianças com autismo, destacam que a presença de uma criança com tal diagnóstico em uma família interfere no funcionamento de todo o grupo familiar, o qual precisa "[...] ajustar seus planos e expectativas futuras às limitações, necessitando adaptar-se e realizar constantes mudanças na sua rotina de vida para atender às necessidades da criança". (EBERT; LORENZINI; FRANCO DA SILVA, 2015, p. 50). Pais de crianças com TEA passam a lidar com uma realidade diferente da idealizada, necessitando desenvolver novas habilidades, a começar pela obtenção de informações sobre o transtorno, conhecimento de recursos e técnicas que possam ajudar a criança no seu desenvolvimento, assim como precisam ser orientadas e receber suporte para se tornarem facilitadores importantes do aprendizado dos filhos.

Marques e Dixe (2011) aplicaram diversos instrumentos para avaliar necessidades da família, adaptação e coesão familiar, ansiedade, depressão e estresse em 50 pais de crianças com autismo e assim levantar as necessidades elencadas por eles. Os resultados indicaram predomínio de organização familiar 
equilibrada, flexível, porém, as famílias que denotaram menor grau de equilíbrio foram as que mencionaram maiores necessidades, solicitando principalmente informação sobre serviços de apoio (médicos, profissionais especializados) e ajuda para discutir problemas e encontrar soluções.

\section{Crenças sobre cuidados parentais e práticas educativas}

As crenças dos pais sobre o que consideram importante para o desenvolvimento dos filhos, comparando com o que de fato fazem, foi alvo de um estudo de Bandeira e Seidl-de-Moura (2012). No estudo, 50 homens e 50 mulheres, tendo ao menos um filho, foram entrevistados. As respostas foram categorizadas em agrupamentos referentes ao tipo de investimento: financeiro, emocional, de cuidados básicos, intelectual, social-espiritual, familiar-pessoal. Ao destacar o investimento idealizado, tanto pais quanto mães valorizaram o emocional, seguido do intelectual. É interessante verificar que, no estudo, os homens, ao descreverem a prática, a atuação direcionada ao âmbito emocional continuou em destaque, corroborando com o que eles mencionaram julgar importante no início da pesquisa. Já as mulheres, que enalteceram o âmbito emocional como o ideal, ao serem questionadas sobre o seu fazer, apontaram para maior engajamento relacionado ao âmbito de cuidados básicos, que tinha ocupado uma das últimas posições na hierarquia materna de prioridades.

Kobarg e Vieira (2008) destacam as crenças como parte da psicologia dos cuidadores, com potencial de interferência no cuidado. Apontam para uma relação dialética entre as crenças e o ambiente físico, ao defender que elas sofrem transformação e transformam o ambiente físico, influenciando o desenvolvimento infantil. Ao avaliar o que as mães valorizavam na educação dos filhos, os autores perceberam que mães da zona urbana, com escolaridade superior, valorizavam mais a estimulação do que as mães da mesma região com baixa escolaridade, as quais destacavam a disciplina. Mães com maior grau de escolaridade, ao valorizarem a estimulação dos filhos, aumentam a probabilidade de engajar-se em ações neste sentido, o que é benéfico para o desenvolvimento da criança.

No que se refere às crenças dos pais sobre o desenvolvimento de seus filhos com deficiências, as pesquisas também são relevantes. Pereira-Silva e Dessen (2007) identificaram as crenças de pais e professores quanto ao desenvolvimento motor, escolarização, profissionalização, relações íntimas e expectativas quanto ao futuro das crianças com Síndrome de Down, comparando com as crenças 
relativas aos mesmos aspectos em famílias com crianças sem deficiência; Stoneman (2007) apresentou algumas diferenças entre pais e mães de criança com Síndrome de Down e deficiência intelectual com etiologia desconhecida, principalmente quanto às crenças parentais; Aguiar (2010) compara crenças de pais e profissionais sobre o desenvolvimento de crianças com Síndrome de Down; Amparo Sobrinho (2010) investiga crenças de pais e influência do contexto; Minetto (2010) analisa a relação entre estresse parental, crenças sobre práticas educativas e funcionamento familiar; Portes (2013) estuda crenças sobre práticas de cuidado e metas de socialização em crianças com Síndrome de Down.

Para Martins et al. (2010, p. 23) "[...] o conjunto de relações estabelecidas entre pais e filhos é permeado por necessidades distintas, porém complementares, como o cuidado, a educação e a promoção do desenvolvimento infantill". Keller (2007) cita seis classes comportamentais (fatores) envolvidas nas práticas de cuidado: cuidado primário; contato corporal; estimulação corporal; estimulação por objetos; contato face a face; envelope narrativo (investimento na linguagem enquanto ação discursiva). Para avaliar as crenças e práticas de cuidados dos pais de filhos com deficiência intelectual, Suizzo (2002) criou a Escala de Crenças Parentais e Práticas de Cuidado (E-CPPC), direcionada para a primeira infância, que toma por base os fatores descritos por Keller.

A E-CPPC permite identificar como os pais acreditam que estão agindo e cuidando das necessidades da criança e orientar os profissionais e os próprios cuidadores em relação ao cuidado da criança. Sobrinho (2010), a partir da E-CPPC, identificou a presença materna significativa, com maior investimento nos cuidados primários que na estimulação. A autora interpreta os achados destacando que os cuidados primários, que estão associados aos cuidados físicos, são tidos como mais cruciais para a sobrevivência do que a estimulação. Portes (2013), utilizando a E-CPPC, constatou diferenças nas crenças entre pais e mães de crianças com Síndrome de Down. As mães tiveram maior tendência a crer na necessidade de prover cuidados primários do que os pais. A autora explicou o resultado considerando o fato de todos os homens pesquisados trabalharem fora e terem como função principal a garantia dos recursos para a prole, enquanto muitas das mães optaram por se dedicar em tempo integral ao cuidado do seu filho com Síndrome de Down. Porém, no que tange às práticas de cuidados primários, os resultados dos pais e mães foi equivalente, ambos atuando no sentido do filho alcançar certa autonomia e independência no futuro.

Será descrito a seguir um estudo que teve por objetivo levantar as crenças e práticas de mães de crianças, na primeira infância, com desenvolvimento atípico, no cuidado de seus filhos. 


\section{Método}

Trata-se de pesquisa descritiva e transversal, que seguiu os procedimentos éticos recomendados. Participaram do estudo 75 mães de crianças com deficiência intelectual, indicadas por escolas regulares de educação infantil e por escolas especiais de uma capital na região sul do país. As mães foram selecionadas a partir do dignóstico das crianças, tendo como referência a Classificação Internacional de Doenças - CID-10 (ORGANIZAÇÃO MUNDIAL DA SAÚDE, 2007), especificamente nos fatores: F70.1 (Retardo mental leve); F71.1 (Retardo mental moderado); e Q90 (Síndrome de Down).

Do total de 75 mães, 41 tinham filhos com diagnóstico de deficiência intelectual (CID-10: F71.1 e F70.1), sem fenótipo (características físicas específicas evidentes), ou seja, com atraso no desenvolvimento, em alguns casos com alterações de comportamento ou características de espectro autista, no entanto, o diagnóstico principal era a deficiência intelectual percebida ao longo dos primeiros anos de vida e não identificada logo quando do nascimento. As outras 34 mães tinham filhos com diagnóstico de Síndrome de Down (CID-10: F71.1; F70.1 e Q90), tendo fenótipo evidente, e com laudo logo quando do nascimento ou nos primeiros dias de vida.

As mães responderam a questionário sociodemográfico, formulado para o presente estudo, com questões referentes à idade, escolaridade, composição familiar, renda familiar, dentre outros aspectos, e à Escala de Crenças Parentais e Práticas de Cuidado (E-CPPC) na primeira infância, de Suizzo (2002), na versão adaptada e validada para a população brasileira por Seidl-de-Moura et al. (2008). O E-CPPC é composto por 50 itens distribuídos em quatro dimensões: Estimulação (chamar a atenção e expor a criança a diferentes estímulos cognitivos e motores); Apresentação apropriada do bebê em público (garantir a apresentação apropriada da criança - social); Responsividade e vínculo ( responder ao filho e criar vínculo com ele e emocional); e Disciplina (educação dos filhos no sentido maior de ensinar regras).

\section{Resultados e discussão}

A idade média das mães foi 38,08 anos ( $\mathrm{DP}=7,93)$, sendo que as mães de crianças com Síndrome de Down $(\mathrm{M}=40,94$ anos, $\mathrm{DP}=6,98)$ eram significati- 
vamente mais velhas que as mães de crianças com Deficiência Intelectual sem fenótipo $(\mathrm{M}=35,71$ anos, $\mathrm{DP}=7,96, \mathrm{p}<0,005)$. Quanto à escolaridade materna, houve certa correspondência entre os dois grupos de mães, com distribuição desde o Ensino Fundamental incompleto até o Ensino Superior completo, porém, com maior concentração de mães com Ensino Médio completo.

O número médio de filhos das famílias participantes foi de 2,03 ( $\mathrm{DP}=1,18)$, sendo que as famílias das crianças com Síndrome de Down tinham em média 2,09 filhos ( $\mathrm{DP}=1,26)$ e as famílias de crianças com Deficiência Intelectual sem fenótipo tinham em média $1,98(\mathrm{DP}=1,13)$ filhos. No que se refere à média salarial, a distribuição dos familiares de acordo com a renda familiar, se convertida em valores percentuais, é bastante próxima nos dois grupos. Segundo dados do Instituto Brasileiro de Geografia e Estatística (IBGE), a renda familiar da população depende da região do país, mas, em 2001, o rendimento mensal per capita brasileiro era de até $1 / 4$ do salário mínimo para $22,9 \%$ das famílias, percentual próximo do encontrado na amostra estudada, já que 15 dos 74 participantes tinham renda mensal familiar de no máximo um salário mínimo.

A idade média das crianças foi de 6,80 anos $(\mathrm{DP}=2,21)$. Quarenta e oito crianças eram do sexo masculino e 27 do sexo feminino, sendo que, destes, 18 meninos e 16 meninas tinham Síndrome de Down. O predomínio do sexo masculino nas crianças sem fenótipo pode estar associado à incidência ligada ao sexo de vários quadros, como nos Transtornos do Espectro Autista, em que há uma proporção de 4,2 nascimentos do sexo masculino para cada criança do sexo feminino nascida. (FOMBONNE, 2009). Das crianças com Síndrome de Down, 94,11\% estavam matriculadas em escola especial, enquanto que, nas crianças com Deficiência Intelectual sem fenótipo, este percentual cai para 80,48\%, índice alto, porém, menor do que o das crianças com Síndrome de Down. O fenótipo pode ser um fator que influencia na inclusão, fazendo com que as crianças que possuem um marcador físico (como a Síndrome de Down) encontrem maiores barreiras para serem incluídas em escolas regulares. Este é um achado que merece estudos futuros, delineados para identificar especificamente tal fator.

As respostas das mães ao questionário sobre Crenças e Práticas Maternas foram sintetizadas na Tabela 1, considerando os fatores Estimulação, Apresentação Apropriada do bebê em público, Responsividade/vínculo e Disciplina. As médias apresentadas foram separadas por família de criança com Síndrome de Down e famílias de crianças com Deficiência Intelectual sem fenótipo. 
TABELA 1 - MÉDIA E DESVIOS DE PADRÕES PARAAS DIMENSÕES DO INSTRUMENTO DE CRENÇAS E PRÁTICAS

\begin{tabular}{lcccc}
\hline Fatores & \multicolumn{2}{c}{$\begin{array}{c}\text { Síndrome de Down } \\
(\mathrm{n}=34)\end{array}$} & \multicolumn{2}{c}{$\begin{array}{c}\text { Deficiência Intelectual } \\
\text { sem fenótipo }(\mathrm{n}=41)\end{array}$} \\
\hline & $\mathrm{M}$ & $\mathrm{DP}$ & $\mathrm{M}$ & $\mathrm{DP}$ \\
Estimulação & 4,02 & 0,66 & 3,98 & 0,46 \\
$\begin{array}{l}\text { Apresentação Apropriada do bebê } \\
\text { em público }\end{array}$ & 4,36 & 0,60 & 4,40 & 0,54 \\
Responsividade e vínculo & 3,47 & 0,55 & 3,59 & 0,62 \\
Disciplina & 3,00 & 0,53 & 3,15 & 0,65 \\
\hline
\end{tabular}

FONTE: As autoras (2015).

Observando a Tabela 1, nota-se que nos dois grupos estudados a dimensão mais valorizada foi a de Apresentação Apropriada do bebê em público, seguida das dimensões Estimulação, Responsividade/vínculo e Disciplina. Considerando que a dimensão Apresentação Apropriada do bebê foi a mais valorizada nos dois agrupamentos, optou-se por estabelecer pares de dimensões, tendo a Apresentação como a variável central, analisando a significância estatística nos dois agrupamentos (crianças com e sem fenótipo). Os resultados podem ser observados na Tabela 2.

TABELA 2 - COMPARAÇÃO EM PARES DA MÉDIA DE APRESENTAÇÃO APROPRIADA DO BEBÊ E DAS MÉDIAS DAS OUTRAS DIMENSÕES DO INSTRUMENTO DE CRENÇAS E PRÁTICAS, RESULTADO DO TEST T PAREADO E SIGNIFICÂNCIA DESSA DIFERENÇA

\begin{tabular}{llll}
\hline Grupo & Pares de médias comparadas & \multicolumn{1}{c}{ T } & \multicolumn{1}{c}{ Sig } \\
\hline \multirow{3}{*}{ Síndrome de Down } & Apresentação e Estimulação & 4,40 & $\mathrm{p}<0,001$ \\
& Apresentação e Responsividade & 8,60 & $\mathrm{p}<0,001$ \\
& Apresentação e Disciplina & 12,08 & $\mathrm{p}<0,001$ \\
\hline \multirow{2}{*}{$\begin{array}{l}\text { Deficiência Intelectual } \\
\text { sem fenótipo }\end{array}$} & Apresentação e Estimulação & 5,04 & $\mathrm{p}<0,001$ \\
& Apresentação e Responsividade & 6,80 & $\mathrm{p}<0,001$ \\
& Apresentação e Disciplina & 13,40 & $\mathrm{p}<0,001$ \\
\hline
\end{tabular}

FONTE: As autoras (2015).

Verifica-se, na Tabela 2, que a média da dimensão Apresentação Apropriada do bebê é maior que as demais e a análise apontou que tal dado tem significância estatística. Isto indica que os pais valorizam mais a dimensão Apresentação do que as demais dimensões. A importância dada à dimensão Apresentação Apropriada do bebê também foi encontrada em outros estudos com crianças sem 
deficiência, bem como a tendência à menor valorização da dimensão Disciplina. (KOBARG; VIEIRA, 2008; SILVA; MAGALHÃES, 2011). Ao mesmo tempo os resultados corroboram com os estudos relacionados a famílias com filhos com deficiência, em que o destaque ficou para os cuidados primários. (SOBRINHO, 2010; PORTES, 2013).

Apesar de alguns estudos com o E-CPPC (KOBARG; VIEIRA, 2008; MARTINS et al., 2010; SILVA; MAGALHÃES, 2011; LORDELO; ROETHLE; MOCHIZUKI, 2012) indicarem correlações com a escolaridade ou renda dos pais com algumas dimensões do instrumento, especialmente a dimensão Disciplina, essa relação não foi encontrada no presente estudo. As correlações encontradas entre as dimensões do instrumento de crenças e práticas são apresentadas na Tabela 3 .

TABELA 3 - CORRELAÇÃO ENTRE AS DIMENSÕES DO INSTRUMENTO DE CRENÇAS E PRÁTICAS

\begin{tabular}{|c|c|c|c|c|c|}
\hline \multirow[t]{2}{*}{ Grupo } & \multicolumn{5}{|c|}{ Correlação } \\
\hline & & Estimulação & $\begin{array}{c}\text { Apresen- } \\
\text { tação }\end{array}$ & $\begin{array}{l}\text { Responsi- } \\
\text { vidade }\end{array}$ & Disciplina \\
\hline \multirow{4}{*}{$\begin{array}{l}\text { Síndrome de } \\
\text { Down }\end{array}$} & 1. Estimulação & 1 & & & \\
\hline & $\begin{array}{l}\text { 2. Apresentação } \\
\text { Apropriada do bebê }\end{array}$ & $0,76^{* *}$ & 1 & & \\
\hline & $\begin{array}{l}\text { 3. Responsividade e } \\
\text { vínculo }\end{array}$ & $0,55 * *$ & $0,46^{* *}$ & 1 & \\
\hline & 4. Disciplina & $0,39 *$ & $0,34 *$ & 0,21 & 1 \\
\hline \multirow{4}{*}{$\begin{array}{l}\text { Deficiência } \\
\text { Intelectual } \\
\text { sem fenótipo }\end{array}$} & 1. Estimulação & 1 & & & \\
\hline & $\begin{array}{l}\text { 2. Apresentação } \\
\text { Apropriada do bebê }\end{array}$ & $0,46^{* *}$ & 1 & & \\
\hline & $\begin{array}{l}\text { 3. Responsividade e } \\
\text { vínculo }\end{array}$ & $0,37 *$ & 0,15 & 1 & \\
\hline & 4. Disciplina & $0,51 * *$ & $0,52 * *$ & 0,19 & 1 \\
\hline
\end{tabular}

${ }^{*} \mathrm{p}<0,05 ; * * \mathrm{p}<0,01$

FONTE: As autoras (2015).

Na comparação das médias das dimensões entre os dois grupos estudados, não houve diferença estatisticamente significativa entre as respostas dadas $(\mathrm{p}>0,05)$. Nos dois grupos não houve correlação entre as dimensões Responsividade/vínculo e Disciplina, ao passo que quanto mais as mães valorizavam a dimensão Estimulação, mais importância davam às dimensões de Apresentação Apropriada do bebê, Responsividade/vínculo e Disciplina, podendo sinalizar que há relação entre a valorização da estimulação de seus filhos com as respostas 
maternas que indicam valorização da apresentação do bebê, o estabelecimento de vínculo produtivo e a colocação de limites no filho. A dimensão Apresentação do bebê também apresentou correlação positiva com a dimensão Disciplina, apontando que quanto mais valor as mães davam à Apresentação, mais valorizavam a Disciplina.

Nas respostas das mães de crianças com Síndrome de Down é possível perceber que a Apresentação Apropriada do bebê foi relacionada positivamente, e com significância estatística, com as dimensões Responsividade/vínculo e Disciplina. Considerando que a dimensão Apresentação Apropriada do bebê foi a mais frequente dentre as dimensões avaliadas pelo instrumento, então, pode-se dizer que este é um aspecto bastante enfatizado por mães de crianças com Síndrome de Down. Porém, é importante retomar Keller (2007), ao afirmar que nenhuma dimensão é mais importante que a outra, mas cada uma delas possui uma função específica no desenvolvimento da criança.

Já nas respostas de mães de crianças com deficiência intelectual sem fenótipo, não foi encontrada correlação estatística entre as dimensões Apresentação Apropriada do bebê e Responsividade/vínculo. Considerando que no grupo de crianças com deficiência intelectual sem fenótipo encontram-se casos de crianças com Transtorno do Espectro Autista, ou outras que apresentam problemas comportamentais intensos, mas muitas vezes fisicamente não apresentam estereótipo que as diferencie das crianças ditas normais, parece que a dimensão Apresentação Apropriada do bebê não fortalece a Responsividade e Vinculação da mãe com a criança, estando a atenção dela possivelmente mais focada na questão comportamental deficitária do filho.

Aguiar (2010) indica que as crenças dos pais sobre a Síndrome de Down estão associadas a afetos que terão por seus filhos (sejam eles positivos ou negativos) e valorização do cuidado, do trabalho e da dedicação despendida a eles. Além disso, para esse autor, tanto os pais como os profissionais atribuem maior valor à aprendizagem e à interação com a criança com Síndrome de Down, do que à maturação biológica, como propulsores do desenvolvimento destas crianças. Com base em tal constatação, é possível hipotetizar que as mães, ao valorizarem a dimensão Apresentação Apropriada da criança com Síndrome de Down, acabam por fortalecer o vínculo e os afetos positivos direcionados aos filhos, o que não pode ser verificado nas respostas das mães de crianças sem fenótipo.

Sobrinho (2010) reforça a importância da rede social de apoio às mães de crianças com Síndrome de Down para o cuidado dos filhos. Para a autora, o fato de grande parte das mães estar ligada a instituições contribui para que elas forneçam cuidados apropriados às suas crianças com Síndrome de Down. Este não foi um aspecto diretamente analisado na pesquisa apresentada, porém, a proximidade dos resultados nos dois grupos de mães pode indicar que ambos 
seriam beneficiados se contassem com rede de apoio. A rede de apoio e o diagnóstico precoce podem ser determinantes nos cuidados oferecidos pelos pais. (MINETTO, 2010).

Para Buscáglia (2006), o aumento do conhecimento sobre a deficiência, a situação atual e futura dos filhos, traz algum conforto aos pais, o que vai refletir em suas crenças, influenciando, consequentemente, nos cuidados oferecidos. Dificuldades podem surgir devido à ideia de que já são crianças limitadas e restritas em muitos aspectos. Para o autor, os pais não as educam como os outros filhos, mas mesmo dentro das limitações, essas crianças precisam de conceitos e limites básicos para a convivência em família e sociedade.

\section{Considerações finais}

Somente o conhecimento promove ações eficientes. A busca constante de informações pode subsidiar profissionais e pais na tarefa de educar. Ter acesso e utilizar informações permite o empoderamento das famílias, propiciando práticas mais efetivas.

A pesquisa desenvolvida mostrou que mães de filhos com deficiência intelectual privilegiam cuidados primários, ou seja, preocupam-se inicialmente com a apresentação apropriada da criança, o que está relacionado a cuidados de higiene, vestimenta, alimentação, dentre outros. Observou-se pequena diferença entre as respostas das mães com filhos com Síndrome de Down em relação às mães de crianças com deficiência intelectual, mas sem fenótipo identificado.

Embora a estimulação do desenvolvimento seja essencial para tais crianças, houve uma tendência em aparecer em segundo plano, especialmente nas respostas do grupo de mães de crianças com déficit intelectual e sem fenótipo. O estudo mostrou que as mães de crianças com Síndrome de Down valorizavam mais a dimensão Estimulação, investiam na Apresentação Apropriada do bebê e tinham diferente índice de Responsividade/vínculo e também de Disciplina, indicando que tais aspectos podem estar relacionados ao fenótipo e à rede de apoio, como associações de pais, sites especializados em informações sobre a síndrome. A criança com déficit cognitivo sem fenótipo tende a demorar mais tempo para ser diagnosticada e por não ter marcadores biológicos reconhecidos quando do nascimento, os pais podem ter mais dificuldade em aceitar o diagnóstico. Esta demora pode atrasar a responsividade em relação aos filhos e, consequentemente, o estabelecimento de vínculo, contando somente mais 
tarde com redes efetivas de apoio, protelando o início de intervenções visando à estimulação apropriada do desenvolvimento.

Por fim, os limites deste estudo incluem o tamanho e perfil da amostra. Novas pesquisas podem ser conduzidas, sendo direcionadas a populações com características socioculturais diferentes, ou ainda, procurando ouvir os pais e as mães e não apenas as mães.

\section{REFERÊNCIAS}

AGUIAR, A. Crenças e conhecimento de pais e profissionais de saúde sobre o desenvolvimento de crianças com sindrome de Down até dois anos de idade. Dissertação (Mestrado) - Programa de Pós-Graduação em Psicologia Social, Universidade do Estado do Rio de Janeiro, Rio de Janeiro, 2010.

AMERICAN PSYCHIATRIC ASSOCIATION (APA). Manual Diagnóstico e Estatístico de Transtornos Mentais. 5. ed. Porto Alegre: Artmed, 2014.

AMPARO SOBRINHO, L. O cuidado a crianças com síndrome de Down sob a perspectiva evolucionista. Dissertação (Mestrado em Psicologia) - Universidade Federal da Bahia, UFBA, Salvador, 2010.

BANDEIRA, T.; SEIDL-DE-MOURA, M. L. Crenças de pais e mães sobre investimento parental. Paidéia, v. 22, n. 53, p. 355-363, 2012.

BRONFENBRENNER, U. A.; MORRIS, P. A. Ecologia do desenvolvimento humano. Tradução: de VERONESE, M. A. V. Porto Alegre: Artes Médicas, 2006.

BUSCAGLIA, L. Os deficientes e seus pais. Rio de Janeiro: Record, 2006.

CALLONERE, A.; FERREIRA ROLIM, S.; HÜBNER, M. M. Relações familiares e escolares em práticas inclusivas. Comportamento em foco, São Paulo, v. 1, p. 87-102, 2011.

CORREA, W.; MINETTO, M. F; KOBREN, R. C.; CUNHA, J. M. Risk Factors for Development of Children with Down Syndrome in Brazil. Creative Education, v. 6, n. 12, p. 1285-1293, 2015.

COUTO, T. H. A. M.; TACHIBANA, M.; AIELLO-VAISBERG, T. M. J. A mãe, o filho e a Síndrome de Down. Paidéia, v. 17, p. 265-272, 2007.

COUTINHO, M. T. B. Apoio à família e formação parental. Análise psicológica, v. 1, n. 22, p. 55-64, 2004.

EBERT, M.; LORENZINI, E.; FRANCO DA SILVA, E. Mães de crianças com transtorno autístico: percepções e trajetórias. Revista Gaúcha de Enfermagem, v. 36, n. 1, p. $49-55,2015$. 
FOMBONNE, E. Epidemiology of pervasive developmental disorders. Pediatic Research, v. 65 , n. 6, p. 591-598, 2009.

FRANCO, V. Introdução à intervenção precoce no desenvolvimento da criança: com a família, com a comunidade, em equipe. Évora: Edições Aloendro, 2015.

GAU, S. S.; CHIU, Y.; SOONG, W.; LEE, M. Parental Characteristics, Parenting Style, and Behavioral Problems Among Chinese Children with Down Syndrome, Their Siblings and Controls in Taiwan. Journal of the Formosan Medical Association, v. 107, n. 9, p. 693-703, 2008.

INSTITUTO BRASILEIRO DE GEOGRAFIA E ESTATÍSTICA (IBGE). Síntese de Indicadores Sociais confirma as desigualdades da sociedade brasileira 2002. Disponível em: <http://www.ibge.gov.br/home/presidencia/noticias/12062003indic2002.shtm>. Acesso em: 30 dez. 2015.

KELLER, H. Cultures of infancy. Mahwah, NJ: Erlbaum, 2007.

KOBARG, A. P. R.; VIEIRA, M. L. Crenças e Práticas de Mães sobre o Desenvolvimento Infantil nos Contextos Rural e Urbano. Psicologia Reflexão e Crítica, v. 21, n. 3, p. 401-408, 2008.

LEAL, E, N. A criança com Síndrome de Down: expectativa da mãe sobre o processo de inclusão escolar. Dissertação (Mestrado) - Faculdade de Filosofia, Ciências e Letras de Ribeirão Preto, Universidade de São Paulo, Ribeirão Preto, 2006.

LORDELO, E. R.; ROETHLE, M.; MOCHIZUKI, A. B. Metas de Socialização em diferentes contextos. Paidéia, v. 22, n. 51, p. 33-42, 2012.

MARQUES, M. H.; DIXE, M. A. R. Crianças e jovens autistas: impacto na dinâmica familiar e pessoal de seus pais. Revista de Psiquiatria Clínica, v. 38, n. 2, p. 66-70, 2011.

MARTINS, G. D. F.; MACARINI, S. M.; VIEIRA, M. L.; SEIDL-DE-MOURA, M. L.; BUSSAB, V. L. R.; CRUZ, R. M. Construção e validação do Inventário de Crenças Parentais sobre Práticas de Cuidado (CPPC) na primeira infância. Psico-USF, v. 15, p. 23-34, 2010.

MATOS, H. S.; ANDRADE, T. S.; MELLO, I. T.; SALES, Z. N. Concepções de mães em relação a filhos portadores de síndrome de down. Revista Saúde e Comportamento, v. 2, n. 1, p. 59-68, 2012.

MINETTO, M. F. Práticas educativas parentais, crenças parentais, estresse parental e funcionamento familiar de pais de crianças com desenvolvimento típico e atípico. Tese (Doutorado) - Universidade Federal de Santa Catarina, Florianópolis, 2010.

MINETTO, M. F.; CREPALDI, M. A.; BRIGAS, M.; MOREIRA, L. C. Práticas Educativas, estresse parental de pais de crianças pequenas com desenvolvimento atípico. Educar em Revista, Curitiba, n. 43, p. 117-132, jan./mar. 2012. 
MINETTO, M. F.; CREPALDI, M. A.; MARTINS, M. Particularidades de Famílias que têm Filhos com Deficiência Intelectual. International Journal of Developmental and Educational Psychology, v. 82, n. 1(1), p. 75-84, 2013.

ORGANIZAÇÃO MUNDIAL DA SAÚDE (OMS). Classificação Estatística Internacional de Doenças e Problemas Relacionados com a Saúde-CID-10. São Paulo, 2008.

PEREIRA-SILVA, N. L.; DESSEN, M. A. Crianças com e sem síndrome de Down: valores e crenças de pais e professores. Revista Brasileira de Educação Especial, v. 13, n. 3, p. 429-446, 2007.

PÉREZ-LÓPEZ, J.; MARTÍNEZ-FUENTES, M. T.; DÍAZ-HERRERO, Á.; NUEZ, A. G. B. Prevención, promoción del desarrollo y atención temprana en la Escuela Infantil. Educar em Revista, Curitiba, n. 43, p. 17-32, 2012.

PORTES, J. M. Crenças sobre práticas de cuidado e metas de socialização de pais e mães com filhos com Síndrome de Down. Tese (Doutorado) - Universidade Federal de Santa Catarina, Florianópolis, 2013.

PRADO, A. F. A. Família e deficiência. In: CERVENY, C. M. O. (Org.). Família e... São Paulo: Ed. Casa do Psicólogo, 2005.

SEIDL-DE-MOURA, M. L.; RIBAS, A. F. P.; SEABRA, K. DA C.; PESSÔA, L.; NOGUEIRA, S. E.; MENDES, D. M. L. F.; ROCHA, S. B.; VICENTE, C. C. Interações mãe-bebê de um e cinco meses de díades urbanas: aspectos afetivos, comportamentos, complexidade e sistemas parentais predominantes. Psicologia Reflexão e Crítica, v. 21, n. 1, p. 66-73, 2008.

SILVA, N. C. B.; AIELLO, A. L. R. Análise descritiva do pai da criança com deficiência mental. Estudos de Psicologia, v. 26, n. 4, p. 493-503, 2009.

SILVA, R. A. M.; MAGALHÃES, C. M. C. Crenças sobre práticas: um estudo sobre mães primíparas de contexto urbano e não-urbano. Revista Brasileira de Crescimento e Desenvolvimento Humano, v. 21, n. 1, p. 39-50, 2011.

SOBRINHO, L. A. O cuidado parental de crianças com síndrome de Down sob a perspectiva evolucionista. Dissertação (Mestrado) - UFBA, Salvador, 2010.

STONEMAN, Z. Examining the down syndrome advantage: Mothers and fathers of young children with disabilities. Journal of Intellectual Disability Research, v. 51, n. 12, p. 1006-1017, 2007.

SUIZZO, M. A. French parents' cultural models and childrearing beliefs. International Journal of Behavioral Development, v. 26, p. 297-307, 2002.

SUNELAITIS, R. C.; ARRUDA, D. C.; MARCON, S. S. A repercussão de um diagnóstico de SD no cotidiano familiar: perspectiva da mãe. Acta Paul Enferm, São Paulo, v. 20, n. 3, p. 264-271, 2007. 
VIEIRA, M. L.; PRADO, A. B. Abordagem evolucionista sobre a relação entre filogênese e ontogênese no desenvolvimento infantil. In: SEIDL-MOURA, M. L. O bebê do século XXI e a psicologia do desenvolvimento. São Paulo: Casa do Psicólogo, 2004.

ZANON, R. B.; BACKES, B.; BOSA, C. A. Identificação dos primeiros sintomas de autismo pelos pais. Psicologia: teoria e pesquisa, v. 30, n. 1, p. 25-33, 2014.

Texto recebido em 21 de janeiro de 2016. Texto aprovado em 21 de janeiro de 2016. 